\title{
Tafenoquine: A Breakthrough Drug for Radical Cure and Elimination of Malaria
}

\author{
Gokul Gopi ${ }^{1}$, Surama Manjari Behera ${ }^{2}$ and Priyamadhaba Behera ${ }^{1 *}$ \\ ${ }^{1}$ All India Institute of Medical Sciences, Bhubaneshwar, India; ${ }^{2}$ Regional Medical Research Center, Bhubaneswar, India
}

\begin{abstract}
Forty percent of the world's population is at risk of Plasmodium vivax infection. Relapse is a feature of malaria caused by $P$. vivax and $P$. ovale due to the presence of the parasite's hypnozoite stage that allows it to stay dormant in the human liver. The associated morbidity and economic burden is high, as $P$. vivax causes severe anemia, miscarriage among pregnant women, malnutrition, and developmental delay in young children due to its chronic relapsing nature. Till recently, for more than 60 years the only licensed antimalarial with proven hypnozoitocidal activity was primaquine. The World Health Organization recommends a regimen of 3-day chloroquine plus 14 days of primaquine for radical cure. Poor adherence to the primaquine course limits its public health benefit on a large scale. Tafenoquine is an 8-aminoquinoline with slower elimination rate, hence a single dose of it is sufficient for hypnozoitocidal activity. Additionally, the schizontocidal activity of tafenoquine makes it a superior drug to the currently available antimalarials, which are mostly single stage specific. Recently, tafenoquine was approved in the USA and Australia for the radical cure of $P$. vivax malaria in patients aged $\geq 16$ years who are receiving appropriate antimalarial therapy for acute $P$. vivax malaria, and for the prophylaxis of malaria in patients aged $\geq 18$ years. We have reviewed the available literature of tafenoquine here, and this article explores the possibility of tafenoquine as a key tool for control and elimination of malaria.
\end{abstract}

\section{Introduction}

Globally, in 2017, an estimated 219 million cases of malaria were reported (95\% confidence interval [CI]: 203-262 million), compared with 239 million cases in 2010 (95\% CI: 219-285 million) and 217 million cases in 2016 (95\% CI: 200-259 million). Fifteen countries within sub-Saharan Africa and India carried nearly $80 \%$ of the global malaria burden. Among those, five countries accounted for nearly $50 \%$ of all malaria cases worldwide: Nigeria (25\%); Democratic Republic of the Congo (11\%); Mozambique (5\%); India (4\%); and Uganda (4\%). ${ }^{1}$

An increasing number of countries are progressing to elimination, with 19 countries attaining elimination status (zero indigenous cases for 3 years or more) between 2000 and 2017. Although

\section{Keywords: Tafenoquine; Malaria; Plasmodium vivax; Primaquine.}

Abbreviations: CI, confidence interval; CQ, chloroquine; CYP2D6, cytochrome P450 2D6; DETECTIVE, Dose and Efficacy Trial Evaluating Chloroquine and Tafenoquine In Vivax Elimination; GATHER, Global Assessment of Tafenoquine Hemolytic Risk; G6PD, glucose 6 phosphate dehydrogenase; TQ, tafenoquine; WRAIR, Walter Reed Army Institute of Research.

Received: January 27, 2019; Revised: May 08, 2019; Accepted: May 18, 2019

*Correspondence to: Priyamadhaba Behera, Bioinformatics Room, Department of Community and Family Medicine, AIIMS, Bhubaneswar - 751019, Odisha, India. Tel: +91 9910830997; E-mail: priya.madhaba@gmail.com

How to cite this article: Gopi G, Behera SM, Behera P. Tafenoquine: A Breakthrough Drug for Radical Cure and Elimination of Malaria. Exploratory Research and Hypothesis in Medicine 2019;4(2):29-34. doi: 10.14218/ERHM.2019.00004. several countries continue to reduce their malaria burden, the rate of reduction has slowed in the highest burden countries; in fact, in some of those countries, malaria cases appear to have risen. With the current global trends being off track for the global technical strategy for malaria 2016-2030 morbidity and mortality targets for 2020 , all indications are that the goals are unlikely to be achieved. To get back onto a trajectory that will ensure the achievement of global technical strategy morbidity and mortality milestones for 2025 , a response is required to change the current trend in countries that are off track, while sustaining the momentum in those that are on target. This calls for intensified efforts, especially in the highest burden countries. ${ }^{1}$

Until recently, Plasmodium vivax was a relatively neglected pathogen, and research and clinical efforts mainly focused on reducing the mortality associated with Plasmodium falciparum. However, $P$. vivax is still a major health and economic burden across Asia and Latin America, where the infection is still prevalent. About $82 \%$ of estimated vivax malaria cases in 2017 occurred in just five countries (India, Pakistan, Ethiopia, Afghanistan and Indonesia). ${ }^{1}$ Unlike $P$. falciparum, $P$. vivax and $P$. ovale have the ability to form hypnozoites that persist in the human liver for variable periods of time and can result in clinical relapses many months or years after the primary infection. Hence, the efforts focused on eradicating malaria need to address this hypnozoite reservoir of $P$. vivax. ${ }^{2}$ A clinical cure of $P$. vivax can be achieved by clearing the blood-borne pathogen from a patient. However, to achieve a radical cure, in addition to the clinical cure, the patient 
has to be cleared of the hypnozoite reservoir so as to prevent any future relapses.

For more than 60 years, primaquine (PQ) has been the only licensed drug for $P$. vivax hypnozoite eradication. ${ }^{3}$ The standard regimen for a radical cure treatment of $P$. vivax infection includes the treatment with artesunate based combination therapy (ACT; including its derivatives) or chloroquine (CQ) for 3 days, along with PQ for 14 days started from day-1 of the treatment. The most significant obstacle to achieving an adequate clinical effectiveness of this regimen is compliance. ${ }^{4}$ Patients often feel asymptomatic within a few days after onset of the treatment, resulting in poor compliance for completing the 14-day course of PQ.

Consider a population as a whole, and this small pool of patients who still carry the dormant parasites act as the pathogen reserve, aiding in the transmission of parasite to other healthy individuals. Level-headed use and forestalling resistance are the key issues with PQ use. PQ has been utilized for over 60 years with foreseen high efficacy in relieving $P$. vivax infection. Its adequacy has been challenged with accessibility, prescribing practices, and adherence.

Dread of hemolytic potential in glucose 6 phosphate dehydrogenase (G6PD)-deficient people fundamentally diminishes eligibility of PQ to be used as a mass administered drug in public health programs. As indicated by the World Health Organization, antimalarial drug resistance emerges because of spontaneous mutation, and thus is unpredictable. Though the mechanism of resistance to CQ has been widely studied and understood, for 8-aminoquinolone compounds like PQ, such a mechanism has not been identified yet. ${ }^{5}$ There has been recently emerging reports mentioning PQ resistance from various parts of the world. Unfortunately, PQ resistance is regularly confused as treatment failure (relapse occurrence), even after the full course of treatment and at the right therapeutic dose. ${ }^{6}$ Along these lines, defining or confirming genuine PQ resistance is disputable.

Baird and Hoffman ${ }^{7}$ performed and audit and published instances of PQ resistance from over more than 30 years past; they showed that an extensive number PQ treatment failure was seen with patients who took $15 \mathrm{mg} /$ day for 5 days. The standard recommended adult dose of PQ is $15 \mathrm{mg}$ /day for 14 days $(210 \mathrm{mg}$ in total). In any case, new studies from a few nations have indicated that this regimen is no longer viable and thus call for higher doses of PQ. ${ }^{5,7}$ Poor compliance, need for increasing drug doses, and rising concerns regarding drug resistance among the malarial parasites have been pushing the scientific community and researchers to search for a drug that could address these concerns and would accelerate the global movement towards eradicating malaria. Tafenoquine (TQ) is a relatively new 8-aminoqunolone, belonging to the same class as PQ with a long elimination half-life (14-28 days versus 4-6 hour for PQ); this long half-life allows infrequent dosing.

\section{History and evolution of the next generation antimalarial: TQ}

Currently available antimalarial drugs have stage-specific activity. Drugs like CQ act mainly on the blood stage of plasmodium and is important for the clinical cure of malaria. Drugs like PQ have a main activity against hypnozoites, thus helping to prevent relapses. Moreover, these drugs have not been successful in breaking the transmission of malarial parasites because of their poor activity against the gametocytic or sporogonic stages of plasmodium. Some of the concerns and liabilities of the current treatment include prolonged treatment duration, increasing reports of resistance, and failing treatments with the need for usage of higher doses and the risk of hemolysis in G6PD-deficient patients. Thus, there is a need for modification in the currently existent treatment protocol for malaria.

The orally active 8-aminoquinoline TQ was discovered in 1978 by scientists at the Walter Reed Army Institute of Research (referred to here as WRAIR), USA during their search for a more convenient alternative to PQ. It is an analogue of PQ and varies only on the presence of a 5-phenoxy group. The prophylactic indication was first developed by GlaxoSmithKline and WRAIR, It was further developed jointly by GlaxoSmithKline and the Medicines for Malaria Venture as a radical cure for P. vivax malaria, while $60^{\circ}$ Pharmaceuticals and WRAIR, in collaboration with the U.S. Army, worked further on prophylaxis of malaria using TQ. ${ }^{8}$

When TQ was first investigated by the U.S. Army, it was as a substitute for PQ that would be more efficacious in a radical cure against relapsing $P$. vivax. Its preclinical developments gained momentum once its potent blood schizontocidal activity against multidrug resistant asexual stages of $P$. falciparum became evident. In January 2013, it was granted the orphan drug status to promote further developments and research on this drug. Further, in December 2013, the U.S. Food and Drug Administration granted a breakthrough therapy designation. ${ }^{9}$ The designation was granted as an attempt to accelerate the development and review times of the drug, since preliminary clinical evidence indicated TQ to represent a substantial improvement over existing therapy. ${ }^{10}$ Finally, a single dose of TQ $300 \mathrm{mg}$ was approved in the USA (July 2018) and Australia (August 2018) for the radical cure of $P$. vivax malaria in patients aged $\geq 16$ years who are receiving appropriate antimalarial therapy for P. vivax malaria; subsequently, in August 2018, TQ $200 \mathrm{mg}$ administered once daily for 3 days followed by once weekly was approved in the USA for the prophylaxis of malaria in patients aged $\geq 18$ years. TQ is under phase 2 development for the same prevention indication in Australia. The safety and efficacy of TQ is not yet established for its use in the pediatric population (as a radical cure in age $<16$ years and as a prophylactic agent in age $<18$ years). A bridging clinical trial (NCT02563496) is currently in phase 2 , and will assess the efficacy of TQ $(50 \mathrm{mg}$ fast dispersible tablet for weight $<35 \mathrm{~kg}$ and adult tablet of $150 \mathrm{mg}$ for children $>35 \mathrm{~kg}$ ) in a pediatric population ( 6 months- 16 years old) of southeast Asia and South America. A brief timeline of important milestones in TQ development is shown in Figure 1.

\section{Pharmacokinetics and pharmacodynamics}

TQ is unique in the sense that it has a very long half-life and, like $\mathrm{PQ}$, is active against the preerythrocytic form (liver), the erythrocytic form (asexual), and the gametocytes of the Plasmodium species, that includes $P$. vivax and $P$. falciparum. ${ }^{11-15}$ TQ exhibits extensive protein binding of over $99.5 \%$ and in a healthy adult has a volume of distribution of $\approx 2,470 \mathrm{~L}$ with an interindividual variability of $24.1 \%$. Though its full excretion profile is unknown, metabolism of TQ is slow. With an apparent oral clearance of $\approx 3 \mathrm{~L} / \mathrm{h}$, it has an average terminal half-life of $\approx 15$ days. While the effect of renal or hepatic impairment on the pharmacokinetics of TQ is still unknown, the pharmacokinetic profile of TQ remains unaffected by age, sex, ethnicity, and bodyweight. Compared with the fasting state, co-administration of TQ with a high-calorie, high-fat meal increased the total exposure to drug by up to $41 \%$ and the time to reach peak plasma concentration was increased by approximately one-third. ${ }^{14}$

Drug interaction studies in healthy volunteers concluded that the pharmacokinetics of TQ were not affected to a clinically relevant extent upon coadministration with $\mathrm{CQ},{ }^{16}$ dihydroartemisinin- 


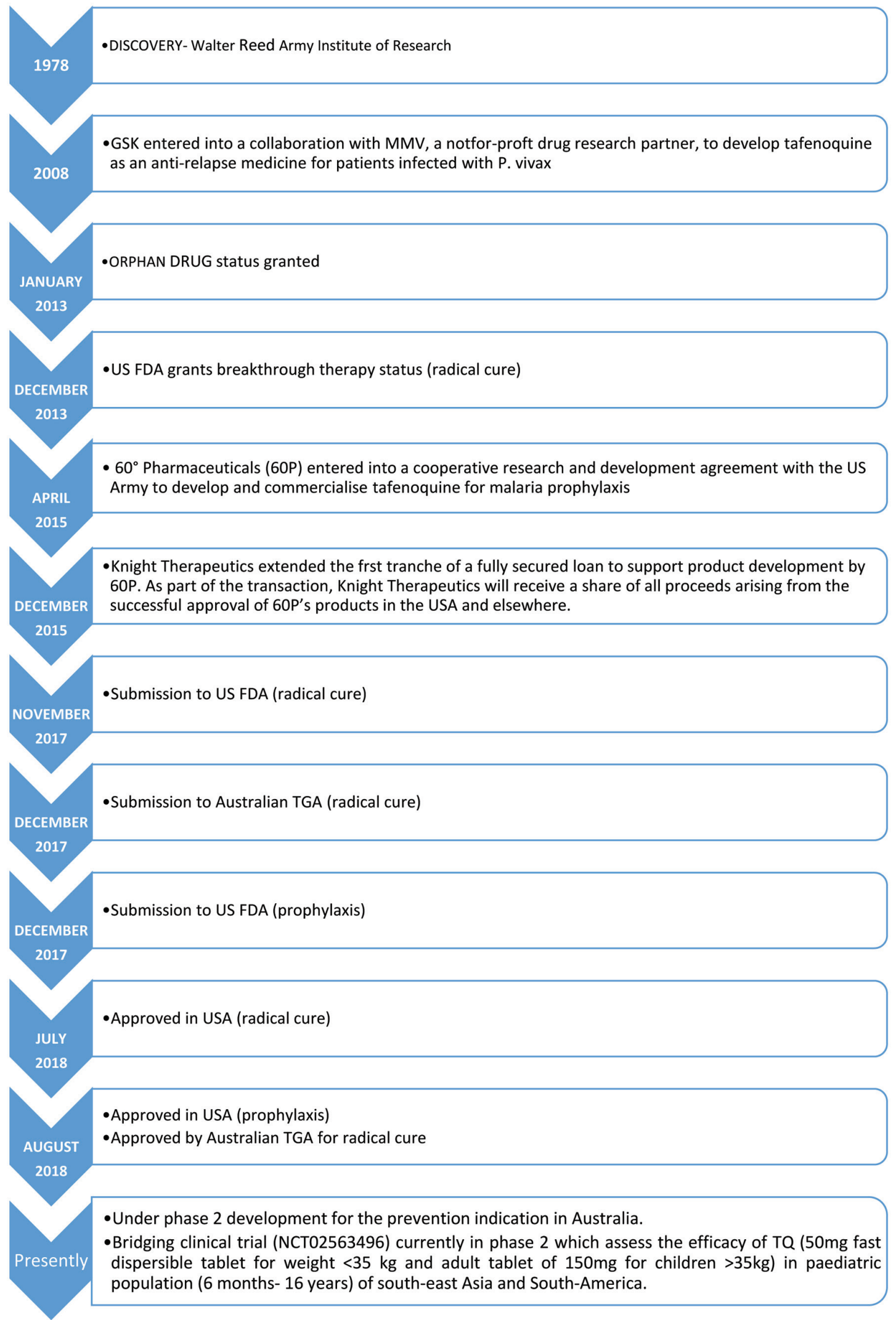

Fig. 1. Important milestones related to Tafenoquine (TQ). 
piperaquine or artemether-lumefantrine. ${ }^{17}$ Correspondingly, TQ did not show a clinically significant effect on the pharmacokinetics of coadministered dihydroartemisinin, piperaquine, artemether. or lumefantrine. ${ }^{17}$

Though the exact mechanism(s) of action responsible for its antiplasmodial activities remain unknown, certain studies involving various protozoan parasites, including $P$. falciparum, have demonstrated TQ to interfere with mitochondrial functions resulting in an apoptotic-like death of the organism ${ }^{18-20}$ TQ may also exert its effect by inhibiting hematin polymerization. ${ }^{21}$ In addition to its antiplasmodial activities, TQ also causes red cell shrinkage and eryptosis or suicidal erythrocyte death, a process similar to apoptosis in nucleated cells. ${ }^{22}$ The activity of TQ targeting the pre-erythrocytic (liver) stages of plasmodium species prevents the development of relapses in $P$. vivax malaria. A study from Thailand focusing on the transmission blocking potential of TQ, evaluated the efficacy of TQ against the sporogonic stage of the $P$. vivax parasite after letting mosquitoes feed on gametocytemic blood containing TQ. TQ reduced the transmission of parasite to the mosquito at doses of $\geq 25 \mathrm{mg} / \mathrm{kg}$. ${ }^{23}$

Like PQ, TQ can induce hemolysis in G6PD-deficient individuals. Drug-induced hemolysis in healthy volunteers with moderately decreased G6PD enzyme activity (40-60\% of normal) and who were heterozygotes for the Mahidol487A G6PD deficiency variant showed a linear correlation with increasing single dose of TQ, from $100 \mathrm{mg}$ through $300 \mathrm{mg}$. However, the hemolytic risk of a single dose TQ $300 \mathrm{mg}$ did not appear to be greater than that with a 14-day course of PQ at $15 \mathrm{mg} /$ day. $^{24}$ Among the heterozygous healthy female volunteers with G6PD enzyme activities of $61-80 \%$ or $>80 \%$ of normal, the greatest drop in hemoglobin following a single-dose TQ of $200 \mathrm{mg}$ were seen in those with lower G6PD enzyme activity levels. ${ }^{24}$ A thorough QT study among 260 healthy volunteers concluded that TQ at therapeutic (300 and 600 $\mathrm{mg})$ and supratherapeutic $(1,200 \mathrm{mg})$ doses did not have a clinically significant effect on cardiac repolarization. ${ }^{25}$

Recent reports have featured a potential pharmacogenetic impact on the adequacy of PQ in people who are normally deficient in cytochrome P450 2D6 (known as CYP2D6) activity. Both in mice and people, this exploration has given reliable proof that metabolic enactment of PQ by CYP2D6 is required for its activity against hypnozoites, perhaps by means of a toxic metabolite. ${ }^{26,27}$ Further research in mice suggested possible extension of this CYP2D liability to other members of the 8-aminoquinolone class, including TQ. ${ }^{28}$

\section{Indications and adverse reactions}

The efficacy of TQ has been demonstrated successfully in prophylaxis as well as radical cure (prevention of relapse) of $P$. vivax malaria. Three key randomized, double-blind, placebo-controlled and/or active referenced, multinational studies have investigated the efficacy of TQ coadministered with CQ as a radical cure for $P$. vivax malaria; these are the Dose and Efficacy Trial Evaluating CQ and TQ In Vivax Elimination (known as DETECTIVE; NCT01376167) parts 1 and 2 and the Global Assessment of TQ Hemolytic Risk (known as GATHER; NCT02216123). ${ }^{29-31}$ Another four randomized, double-blinded, placebo-controlled and/or active referenced studies have evaluated the prophylactic efficacy of TQ (200 mg for 3 days followed by weekly $200 \mathrm{mg}$ maintenance doses); one phase III trial conducted on healthy Australian soldiers (nonimmune subjects) deployed in a malaria endemic zone of Timor Leste and three phase II trials conducted on inhabitants of African regions endemic for $P$. falciparum malaria trials
(NCT02488980, NCT02491606, and NCT0248890)..$^{32-35}$

Though TQ was generally well tolerated in the clinical trials, some of the most common adverse events reported were headache, dizziness, nausea, vomiting, and decreased hemoglobin. In the DETECTIVE part 2 trial, the most common (incidence $\geq 5 \%$ ) adverse reaction reported prior to day 29 among the TQ plus CQ recipients was dizziness ( $8 \%$ vs. $3 \%$ with CQ alone) followed by nausea $(6 \%$ vs. $7 \%$ ), vomiting ( $6 \%$ vs. $5 \%)$, decreased hemoglobin $(5 \%$ vs. $2 \%)$ and headache $(5 \%$ vs. $7 \%) .{ }^{15}$ Other adverse reactions, like neuropsychiatric disorders (anxiety, insomnia, abnormal dreams), abnormal blood biochemical panel (raised blood creatinine, increased blood methemoglobin, increased alanine aminotransferase), and eye disorders (photophobia, vortex keratopathy) were reported in $\leq 3 \%$ of clinical trial subjects who received a single dose of 300 mg TQ. ${ }^{15}$ The concerns with ophthalmic safety of single-dose 300 $\mathrm{mg}$ TQ was further analyzed in a dedicated ophthalmologic study involving nearly 300 healthy volunteers (NCT02658435). There were no reports of vortex keratopathy or retinal abnormalities associated with TQ in this study. ${ }^{36,37}$

Schmidt et al. ${ }^{38}$ have shown that the tissue schizontocidal activity of PQ is a function of total dose rather than of duration of administration, but the toxicity of PQ limits the amount patients may be given in any period of time. Additionally, the fact that the therapeutic dose and the toxic dose are close, leads to serious problems associated with the use of PQ. Clinically important side-effects of PQ include gastrointestinal disturbances, methemoglobinemia, acute intravascular hemolysis in individuals deficient in G6PD enzyme, and possible immunosuppression through inhibition of lymphocyte proliferation. ${ }^{39}$

The main safety concern with 8-aminoquinolines like PQ and TQ is drug-induced hemolysis in patients with G6PD enzyme deficiency. ${ }^{36}$ The DETECTIVE trials excluded patients with $<70 \%$ of normal G6PD activity. The GATHER study assessed the hemolytic potential of TQ (300 $\mathrm{mg}$ as single dose), which excluded male patients with $<70 \%$ of normal G6PD activity and female patients with $<40 \%$ of normal G6PD activity. ${ }^{31}$ Clinically relevant hemolysis was defined as an overall drop in hemoglobin to $<6.0 \mathrm{~g} / \mathrm{dL}$ or a decrease in hemoglobin of $\geq 30 \%$ or $>3 \mathrm{~g} / \mathrm{dL}$ from baseline at any visit after the first dose of study medication. ${ }^{31}$ Only 4 of the 166 participants $(2.4 \%$; $95 \%$ CI: 0.9 to 6.0$)$ who received TQ plus CQ experienced clinically significant hemolysis up to day 180 (one of the coprimary endpoints) versus 1 of 85 participants $(1.2 \%$; $95 \%$ CI: 0.2 to 6.4 ) who received PQ plus CQ. The other coprimary endpoint (prespecified) was the proportion of females who experienced clinically relevant hemolysis up to day 180 who also had moderate G6PD deficiency (G6PD enzyme activity between 40 $70 \%$ ). However, in that event the single patient who was enrolled did not receive TQ. ${ }^{31}$

\section{Public health importance}

The current global strategies for eliminating malaria include measures for prevention of malaria, early detection of the disease with early initiation of appropriate treatment, and active surveillance systems. Some of the challenges faced by $P$. vivax malaria-endemic countries include limited access to effective drugs treating liver stages of the parasite (schizonts and hypnozoites), emergence of drug resistance, and misperception of $P$. vivax malaria as nonlethal. On average, as shown from experience, the elimination of $P$. vivax foci can be achieved but not in $<3$ years, compared with the elimination of $P$. falciparum, which can be achieved in 1 year. ${ }^{40}$ One of the important steps toward malaria elimination is to achieve 
radical cure among all patients with $P$. vivax malaria. Currently, the treatment for an uncomplicated $P$. vivax infection consists of drug therapy lasting 14 days ( 3 days CQ/artesunate based combination therapy with 14 days PQ). This long therapy duration, even after rendering the patient asymptomatic, is one of the most challenging obstacles, as it is often the most common reason for poor compliance. The poor adherence to PQ limits its public health benefit on a large scale. With the current developments and evidence suggesting the equal or even better efficacy of TQ over the existing treatment regimen, it seems to be an ideal candidate for tackling the problem of noncompliance due to its single dosing. Moreover, TQ has been shown to be effective even against some CQ-resistant strains. Another important benefit of TQ is in its potential to cut off transmission of the parasite within a community, due to its activity against the sporogonic stage of the $P$. vivax parasite. ${ }^{20}$ Hence, such a drug, if introduced in a highly endemic region, would show promising results in decreasing the disease burden.

\section{Future research directions}

PQ has been in consistent use since 1952 for preventing relapse in patients with $P$. vivax and $P$. ovale malaria. Hemolysis is the major concerning side effect of PQ in individuals who are deficient in G6PD enzyme or its activity. TQ seems to address the problem of compliance due to its single dosing regimen and seems to be an ideal candidate to replace PQ; however, it has similar hemolysis potential as PQ. The available data related to hemolysis and pharmacologic profile of PQ use in community can be utilized for the use of TQ since both the drugs are from the same family and have similar pharmacological properties.

Recently, there have been advancements in the technological community to address the issue of hemolysis and different tools and sensors have been developed to assess the G6PD enzyme activity. Though with emerging technologies and feedback from the use of these devices, the scientific community has been driven to reduce the size and complexity of these devices; yet, they still, by far, are not portable and the operation of these devices requires trained technicians, which largely limits use in community health programs. Recently, a new device - STANDARD TM G6PD - has been developed by a south Korean company (SD BIOSENSOR) with support from PATH. This is a handheld device that delivers results in 2 minutes and provides a quantitative measure of G6PD activity, including in heterozygous women, by using a capillary blood sample and a cartridge similar in format to a glucose meter. According to the company's description, "It provides a quantitative measurement of both G6PD levels and total hemoglobin, enabling health workers to determine if radical cure with an 8-aminoquinoline-based drug is appropriate for patients." ${ }^{41}$ The product is currently Conformité Européenne-marked to conform with the European Union In Vitro Diagnostic Medical Devices Directive (98/79/EC), with ongoing clinical evaluation through studies in Brazil, Ethiopia and India and a full clinical evaluation report is expected by mid-2019.41

Further research about the availability of a low-cost, portable and efficient point-of-care testing platform for detection of G6PD deficiency, better availability and affordability, as well as more detailed risk versus benefit data regarding the use of TQ will be vital before its use in public health care for malaria control.

\section{Conclusions}

TQ is a promising new drug for advancement of the goal towards global malaria elimination. Currently, TQ is approved in the USA and Australia for the radical cure of $P$. vivax malaria in patients aged $\geq 16$ years who are receiving appropriate antimalarial therapy for acute $P$. vivax malaria and for the prophylaxis of malaria in patients aged $\geq 18$ years. Similar efficacy to primaquine with a single dosing regimen, with better compliance, along with the recent development of small portable devices to quantitatively analyze G6PD activity, makes TQ a promising breakthrough drug for radical cure of malaria. Further research about the availability of a low-cost, portable and efficient point-of-care testing platform for detection of G6PD deficiency, as well as more detailed risk versus benefit data regarding the use of TQ, will be vital before its widespread use in public health care.

\section{Conflict of interest}

The authors declare they have no conflict of interests.

\section{Author contributions}

Development of concept (PB, SMB and GG), manuscript writing (GG and $\mathrm{PB}$ ), review of manuscript (SMB, GG and $\mathrm{PB}$ ).

\section{References}

[1] WHO | World malaria report 2018. WHO [Internet]. 2018 [cited 2019 Jan 23]; Available from: https://www.who.int/malaria/publications/ world-malaria-report-2018/report/en/.

[2] Wells TNC, Burrows JN, Baird JK. Targeting the hypnozoite reservoir of Plasmodium vivax: the hidden obstacle to malaria elimination. Trends Parasitol 2010;26(3):145-151. doi:10.1016/j.pt.2009.12.005.

[3] Vale N, Moreira R, Gomes P. Primaquine revisited six decades after its discovery. Eur J Med Chem 2009;44(3):937-953. doi:10.1016/j. ejmech.2008.08.011.

[4] John GK, Douglas NM, Von Seidlein L, Nosten F, Baird JK, White NJ, et al. Primaquine radical cure of Plasmodium vivax: A critical review of the literature. Malar J 2012;11:280. doi:10.1186/1475-2875-11-280.

[5] Buchachart K, Krudsood S, Singhasivanon P, Treeprasertsuk S, Phophak N, Srivilairit S, et al. Effect of primaquine standard dose ( $15 \mathrm{mg} /$ day for 14 days) in the treatment of vivax malaria patients in Thailand. Southeast Asian J Trop Med Public Health 2001;32(4):720726.

[6] Recht J, White NJ, Ashley E, World Health Organization, Mahidol Oxford Research Unit. Safety of 8-Aminoquinoline antimalarial medicines. Geneva, WHO. 2015.

[7] Baird JK, Hoffman SL. Primaquine therapy for malaria. Clin Infect Dis 2004;39(9):1336-1345. doi:10.1086/424663.

[8] Tafenoquine - GlaxoSmithKline/60 Degrees Pharmaceuticals - AdisInsight [Internet]. [cited 2019 Jan 20]. Available from: https://adisinsight.springer.com/drugs/800003404.

[9] GSK and MMV announce FDA Breakthrough Therapy designation for tafenoquine for Plasmodium vivax malaria | GSK [Internet]. [cited 2019 Jan 22]. Available from: https://us.gsk.com/en-us/media/pressreleases/2013/gsk-and-mmv-announce-fda-breakthrough-therapydesignation-for-tafenoquine-for-plasmodium-vivax-malaria/.

[10] Breakthrough therapy designation: Exploring the qualifying criteria [Internet]. Available from: https://www.brookings.edu/events/ breakthrough-therapy-designation-exploring-the-qualifying-criteria/.

[11] Crockett M, Kain KC. Tafenoquine: a promising new antimalarial agent. Expert Opin Investig Drugs 2007;16(5):705-715. doi:10.1517/13543784.16.5.705.

[12] Campo B, Vandal O, Wesche DL, Burrows JN. Killing the hypnozoitedrug discovery approaches to prevent relapse in Plasmodium vivax. 
Pathog Global Health 2015;109(3):107-122. doi:10.1179/204777321 $5 Y .0000000013$.

[13] Ebstie YA, Abay SM, Tadesse WT, Ejigu DA. Tafenoquine and its potential in the treatment and relapse prevention of Plasmodium vivax malaria: The evidence to date. Drug Des Devel Ther 2016;10:23872399. doi:10.2147/DDDT.S61443.

[14] Fda. Highlights of Prescribing Information-Arakoda ${ }^{\mathrm{TM}}$ [Internet]. [cited 2019 Jan 20]. Available from: https://www.fda.gov/media/114755/ download.

[15] Fda, cder. Highlights of Prescribing Information-Krintafel [Internet]. [cited 2019 Jan 20]. Available from: https://www.accessdata.fda. gov/drugsatfda_docs/label/2018/210795s000lbl.pdf.

[16] Miller AK, Harrell E, Ye L, Baptiste-Brown S, Kleim JP, Ohrt C, et al. Pharmacokinetic interactions and safety evaluations of coadministered tafenoquine and chloroquine in healthy subjects. $\mathrm{Br} \mathrm{J}$ Clin Pharmacol 2013;76(6):858-867. doi:10.1111/bcp.12160.

[17] Green JA, Mohamed K, Goyal N, Bouhired S, Hussaini A, Jones SW, et al. Pharmacokinetic interactions between tafenoquine and dihydroartemisinin-piperaquine or artemether-lumefantrine in healthy adult subjects. Antimicrob Agents Chemother 2016;60(12):73217332. doi:10.1128/AAC.01588-16.

[18] Lanners HN. Effect of the 8-aminoquinoline primaquine on culturederived gametocytes of the malaria parasite Plasmodium falciparum. Parasitol Res 1991;77(6):478-481. doi:10.1007/BF00928413.

[19] Carvalho L, Luque-Ortega JR, Manzano Jl, Castanys S, Rivas L, Gamarro F. Tafenoquine, an antiplasmodial 8-aminoquinoline, targets Leishmania respiratory complex III and induces apoptosis. Antimicrob Agents Chemother 2010;54(12):5344-5351. doi:10.1128/ AAC.00790-10.

[20] Carvalho L, Martínez-García M, Pérez-Victoria I, Manzano JI, Yardley $\mathrm{V}$, Gamarro F, et al. The oral antimalarial drug tafenoquine shows activity against Trypanosoma brucei. Antimicrob Agents Chemother 2015;59(10):6151-6160. doi:10.1128/AAC.00879-15.

[21] Vennerstrom JL, Nuzum EO, Miller RE, Dorn A, Gerena L, Dande PA, et al. 8-Aminoquinolines active against blood stage Plasmodium falciparum in vitro inhibit hematin polymerization. Antimicrob Agents Chemother 1999;43(3):598-602. doi:10.1128/AAC.43.3.598.

[22] Al Mamun Bhuyan A, Bissinger R, Stockinger K, Lang F. Stimulation of Suicidal Erythrocyte Death by Tafenoquine. Cell Physiol Biochem 2016;39(6):2464-2476. doi:10.1159/000452514.

[23] Ponsa N, Sattabongkot J, Kittayapong P, Eikarat N, Coleman RE. Transmission-blocking activity of tafenoquine (WR-238605) and artelinic acid against naturally circulating strains of plasmodium vivax in Thailand. Am J Trop Med Hyg 2003;69(5):542-547. doi:10.4269/ ajtmh.2003.69.542.

[24] Rueangweerayut R, Bancone G, Harrell EJ, Beelen AP, Kongpatanakul S, Möhrle JJ, et al. Hemolytic potential of tafenoquine in female volunteers heterozygous for Glucose-6-Phosphate Dehydrogenase (G6PD) Deficiency (G6PD Mahidol Variant) versus G6PD-Normal volunteers. Am J Trop Med Hyg 2017;97(3):702-711. doi:10.4269/ ajtmh.16-0779.

[25] Green JA, Patel AK, Patel BR, Hussaini A, Harrell EJ, McDonald MJ, et al. Tafenoquine at therapeutic concentrations does not prolong fridericia-corrected QT interval in healthy subjects. J Clin Pharmacol 2014;54(9):995-1005. doi:10.1002/jcph.302.

[26] Pybus BS, Sousa JC, Jin X, Ferguson JA, Christian RE, Barnhart R, et al. CYP450 phenotyping and accurate mass identification of metabolites of the 8-aminoquinoline, anti-malarial drug primaquine. Malar J 2012;11:259. doi:10.1186/1475-2875-11-259.

[27] Joan Ingram RH, Crenna-Darusallam C, Soebianto S, Noviyanti R, Kevin Baird J. The clinical and public health problem of relapse de- spite primaquine therapy: Case review of repeated relapses of Plasmodium vivax acquired in Papua New Guinea. Malar J 2014;13:488. doi:10.1186/1475-2875-13-488.

[28] Vuong C, Xie LH, Potter BMJ, Zhang J, Zhang P, Duan D, et al. Differential Cytochrome P450 2D Metabolism Alters Tafenoquine Pharmacokinetics. Antimicrob Agents Chemother 2015;59(7):3864-3849. doi:10.1128/AAC.00343-15.

[29] Llanos-Cuentas A, Lacerda M V, Rueangweerayut R, Krudsood S, Gupta SK, Kochar SK, et al. Tafenoquine plus chloroquine for the treatment and relapse prevention of Plasmodium vivax malaria (DETECTIVE): A multicentre, double-blind, randomised, phase $2 \mathrm{~b}$ doseselection study. Lancet 2014;383(9922):1049-1058. doi:10.1016/ S0140-6736(13)62568-4.

[30] US National Institutes of Health. ClinicalTrials.gov identifier NCT01376167. 2018. [Internet]. Available from: https://clinicaltrials. gov/ct2/show/NCT01376167.

[31] US National Institutes of Health. ClinicalTrials.gov identifier NCT02216123. 2018. [Internet]. Available from: https://clinicaltrials. gov/ct2/show/NCT02216123?term=NCT02216123\&rank=1.

[32] Novitt-Moreno A, Ransom J, Dow G, Smith B, Read LT, Toovey S. Tafenoquine for malaria prophylaxis in adults: An integrated safety analysis. Travel Med Infect Dis 2017;17:19-27. doi:10.1016/j. tmaid.2017.05.008.

[33] Nasveld PE, Edstein MD, Reid M, Brennan L, Harris IE, Kitchener SJ, et al. Randomized, double-blind study of the safety, tolerability, and efficacy of tafenoquine versus mefloquine for malaria prophylaxis in nonimmune subjects. Antimicrob Agents Chemother 2010;54(2):792-798. doi:10.1128/AAC.00354-09.

[34] Dow GS, McCarthy WF, Reid M, Smith B, Tang D, Shanks GD. A retrospective analysis of the protective efficacy of tafenoquine and mefloquine as prophylactic anti-malarials in non-immune individuals during deployment to a malaria-endemic area. Malar J 2014;13:49. doi:10.1186/1475-2875-13-49.

[35] Dow GS, Liu J, Lin G, Hetzell B, Thieling S, McCarthy WF, et al. Summary of anti-malarial prophylactic efficacy of tafenoquine from three placebo-controlled studies of residents of malaria-endemic countries. Malar J 2015;14:473. doi:10.1186/s12936-015-0991-x.

[36] Tafenoquine Tablet, $150 \mathrm{mg}$ Meeting of the Antimicrobial Drugs Advisory Committee (AMDAC) [Internet]. 2018 [cited 2019 Jan 20]. Available from: https://www.fda.gov/downloads/advisorycommittees/committeesmeetingmaterials/drugs/anti-infectivedrugsadvisorycommittee/ucm612874.pdf.

[37] Assessment of any potential retinal effects of tafenoquine (TQ) - Full Text View - ClinicalTrials.gov [Internet]. [cited 2019 Jan 20]. Available from: https://clinicaltrials.gov/ct2/show/NCT02658435?term $=\% 28 \mathrm{~N}$ CT02658435\%29\&rank; $=1$.

[38] Vaughan D, Schmidt LH, Rasco J, Fradkin R. Radical cure of infections with Plasmodium cynomolgi: a function of total 8-aminoquinoline dose. Am J Trop Med Hyg 1977;26(6 Pt 1):1116-1128. doi:10.4269/ ajtmh.1977.26.1116.

[39] Clyde DF. Clinical problems associated with the use of primaquine as a tissue schizontocidal and gametocytocidal drug. Bull World Health Organ 1981;59(3):391-395.

[40] PAMPANA E. A textbook of malaria eradication. 2nd edition. [Internet]. A textbook of malaria eradication. 2nd edition. London: Oxford University Press.; 1969 [cited 2019 Jan 23]. Available from: https:// www.cabdirect.org/cabdirect/abstract/19702900209.

[41] PATH and SD BIOSENSOR announce partnership to advance diagnostic test critical for malaria treatment and elimination I PATH [Internet]. [cited 2019 Apr 16]. Available from: https://www.path.org/ media-center/path-and-sd-biosensor-announce-partnership/. 